\title{
MÔ HÌNH HOÁ BỀ MặT CÔNG TRİNH TÙ DŨ̃ LIỆU ĐO CỦA MÁY TOÀN ĐẠC ĐIỆN TỬ TRIMBLE S6 VỚI CHẾ ĐỘ ĐO KHÔNG GƯƠNG
}

TS. ĐINH CÔNG HÒA, ThS. HOÀNG TH!̣ THỦY

Trường Đại học Mỏ - Địa chất

\section{1. Đặt vấn đề}

Để đánh giá chất lượng công trình, ngoài tiêu chí an toàn, bền vững cần quan tâm đến một số yêu cầu kỹ thuật khác như kích thước và hình dáng của công trình so với thiết kế của nó. Cũng có thể coi các yêu cầu này là tiêu chuẩn hình học và thẩm mỹ của công trình.

Việc áp dụng các tiến bộ của khoa học công nghệ vào sản xuất là một yêu cầu cấp thiết, nhằm nâng cao năng suất lao động và góp phần tự động hoá quá trình sản xuất. Công nghệ điện tử-tin học đã và đang được ứng dụng rộng rãi trong mọi lĩnh vực của đời sống xã hội. Trong Trắc địa, công nghệ điện tử-tin học cũng đã được ứng dụng rộng rãi. Các máy toàn đạc điện tử độ chính xác cao, máy thu GPS và các phần mềm tiện ích... đã và đang dần dần thay thế các loại máy quang học và các phương pháp đo đạc cổ truyền với độ chính xác không cao, năng suất lao động thấp.

Nội dung bài báo nghiên cứu quy trình đo và trút số liệu bằng máy toàn đạc điện tử (TĐĐT) Trimble S6 với chế độ đo không gương, xây dựng chương trình xác định độ thẳng đứng, độ phẳng tường nhà cao tầng và mô hình hoá bề mặt này theo số liệu đo đạc.

\section{Nội dung}

a. Giới thiệu máy toàn đạc Trimble S6 với chế độ đo không gương.
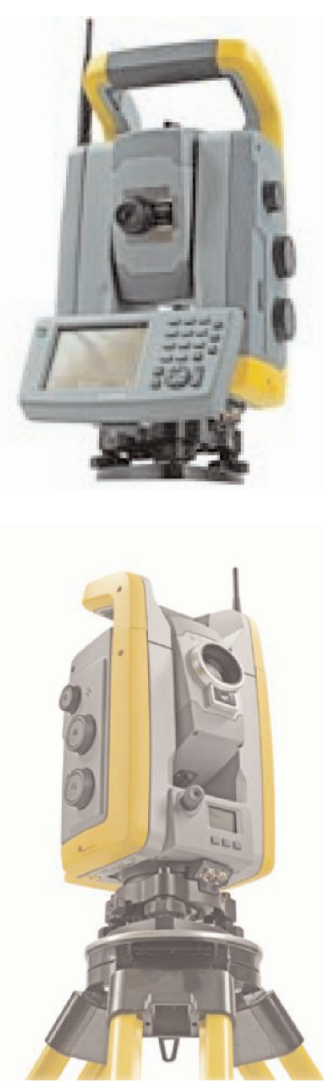

Hình 1: Máy toàn đạc điện tử Trimble S6

Trimble S6 là máy toàn đạc điện tử đo theo chế độ Robotic (tự động bắt mục tiêu) nên sẽ giúp nâng cao năng suất công việc. Trimble S6 với rất nhiều đặc tính mới và công nghệ tiên tiến sẽ mang đến nhiều giá trị tiện ích. Ngoài ra, Trimble S6 cũng giải quyết hầu hết các yêu cầu của công tác trắc địa, có khả năng làm việc cùng với chế độ đo GPS hay quét 3D theo mô hình dưới đây 


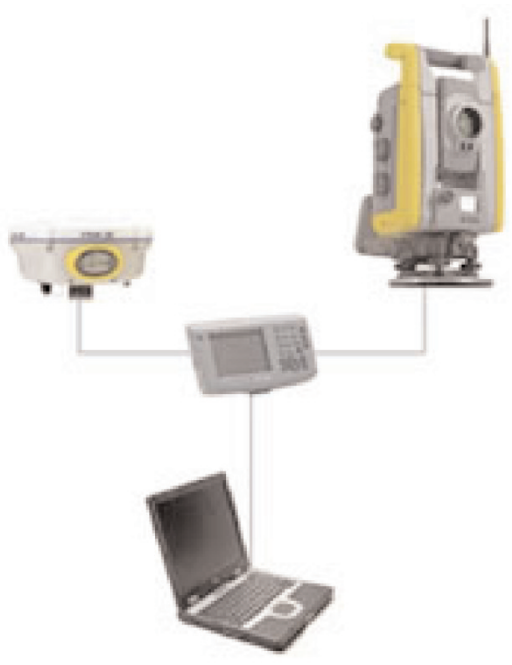

Hình 2: Kết hợp giữa máy S6 với máy GPS và Máy tính

b. Phương pháp mô hình hóa bề mặt công trình

Để mô hình hoá bề mặt công trình theo các số liệu đo đạc, cần sử dụng hàm toán học biểu diễn mặt rồi xấp xỉ tập hợp các điểm quan trắc theo hàm đã chọn.

Chúng ta đã biết, bề mặt công trình thực tế rất phức tạp, biến thiên không theo một quy luật toán học nào. Do đó, có thể thấy rằng không có một hàm toán học nào (bề mặt toán học) có thể trùng khít với mọi điểm trên bề mặt thực tế. Vì vậy, khi dùng một bề mặt toán học nào đó để mô phỏng bề mặt công trình, cần chia đối tượng ra từng khu vực nhỏ sao cho bề mặt của từng khu vực nhỏ được mô phỏng phù hợp nhất bởi hàm toán học đã chọn.

Việc mô phỏng bề mặt công trình cần phải thoả mãn điều kiện "tổng bình phương độ lệch của các điểm trên bề mặt toán học so với các điểm tương ứng trên bề mặt công trình là nhỏ nhất". (Xem hình 3)

Số lượng điểm đo phụ thuộc vào số lượng tham số cần xác định của hàm toán học dùng để xấp xỉ bề mặt. Nếu số lượng điểm đúng bằng số lượng các tham số thì ở các điểm đo bề mặt toán học trùng khít với bề mặt công trình. Khi số điểm đo trực tiếp nhiều hơn cố lượng các tham số thì bài toán sẽ được giải theo phương pháp số bình phương nhỏ nhất theo trình tự như sau:

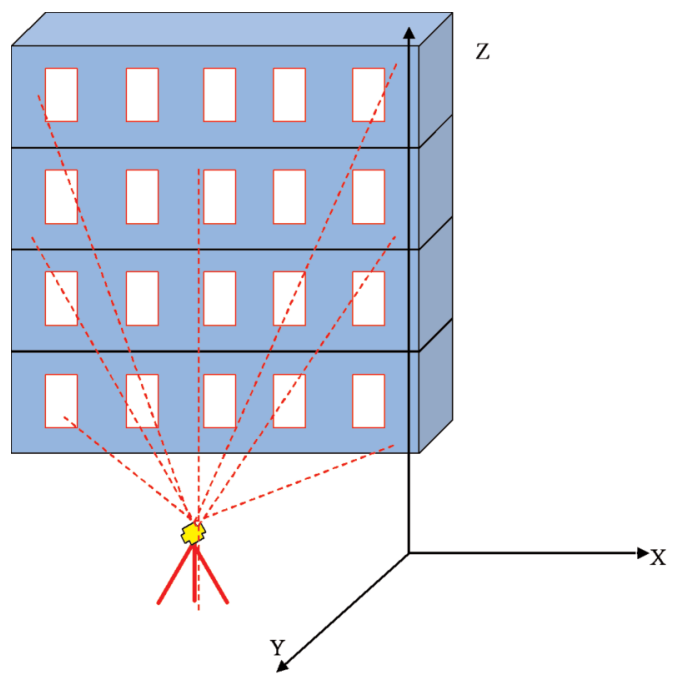

Hình 4: Xác định độ nghiêng và phẳng của tường nhà cao tầng

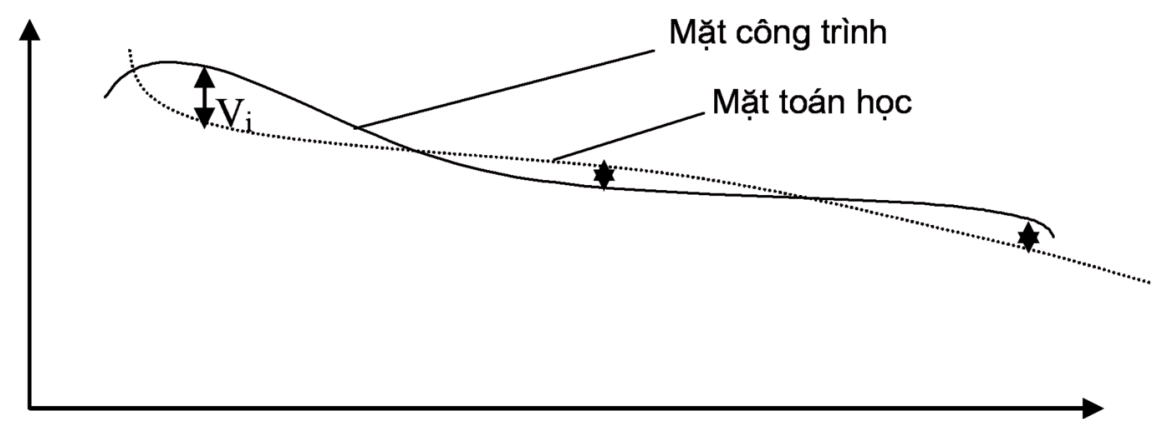

Hình 3: Bài toán xây dụng mô hình 
Bước 1: Từ tọa độ $(X, Y, Z)$ của các điểm lập phương trình mặt phẳng xấp xỉ:

$$
a \cdot X+b . Y+c . Z+d=0
$$

Trong đó: $a, b, c, d$ là các tham số mặt phẳng cần phải xác định.

Bước 2: Lập hệ phương trình số hiệu chỉnh theo công thức:

$$
V=A Q+L
$$

Trong đó: $A$ - ma trận hệ số; $Q$ - vector các tham số của mặt phẳng

$$
\mathrm{L} \text { - véc tơ số hạng tự do }
$$

Bước 3: Lập hệ phương trình chuẩn:

$$
A^{\top} A Q+A^{\top} L=0
$$

Bước 4: Giải hệ phương trình chuẩn nhận được các tham số mặt phẳng

Bước 5: Tính cosin các góc chỉ phương của vector vuông góc mặt phẳng

Bước 6: Tính toán các kết quả:

- Tính độ nghiêng của tường (góc hợp bởi trục thẳng đứng $Z$ và mặt phẳng).

- Tính khoảng cách từ các điểm đo đến mặt phẳng theo công thức

$$
\delta_{1}=X_{i} \cdot \cos \alpha+Y_{i} \cdot \cos \beta+Z_{i} \cdot \cos \gamma-p
$$

Trong đó: $\mathrm{X}_{\mathrm{i}}, \mathrm{Y}_{\mathrm{i}}, \mathrm{Z}_{\mathrm{i}}$ là tọa độ của điểm đo;

$p$ là khoảng cách từ gốc tọa độ đến mặt phẳng.

- Giá trị lồi lõm cực đại:

$$
\Delta_{\mathrm{i}}=\delta_{\mathrm{I}, \operatorname{Max}}-\delta_{\mathrm{I}, \mathrm{Min}}
$$

- Lập tỷ số thể hiện độ không phẳng tương đối.

Độ nghiêng, độ lồi lõm cực đại, độ không phẳng tương đối của tường tính từ các tham số mặt phẳng sẽ phản ánh khách quan chất lượng hình học của bức tường.

c. Thực nghiệm

Trong phần thực nghiệm, chúng tôi đã xây dựng một modul chương trình tính toán độ nghiêng và độ phẳng tường nhà cao tầng theo các thuật toán nêu trên theo các dữ liệu đo không gương từ máy Trimble S6. Modul này có các chức năng sau:

- Tính độ nghiêng của công trình;

- Tính độ lồi Iõm cực đại;

- Tính độ không phẳng tương đối của tường.

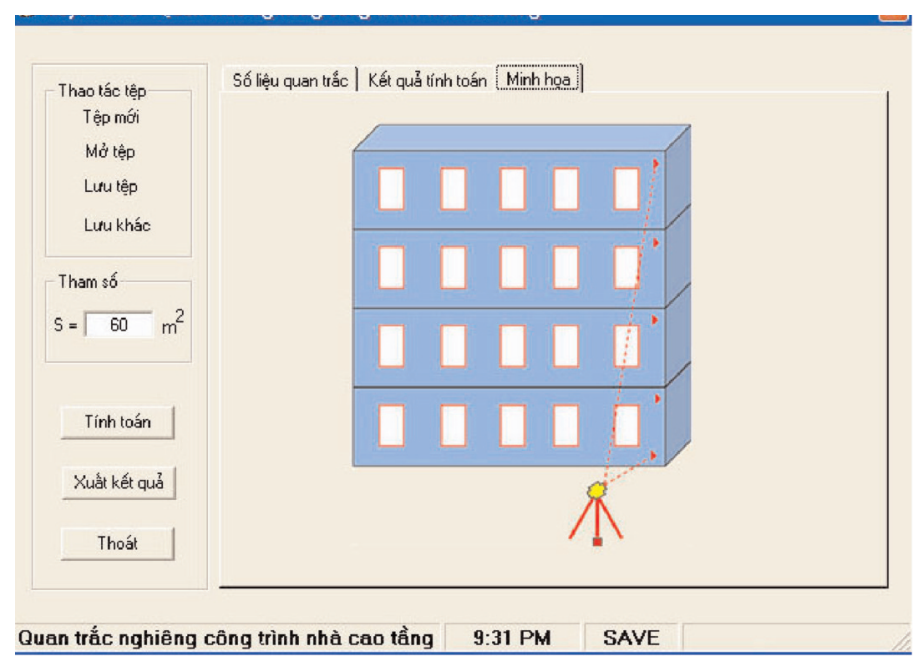

Hình 5: Giao diện đo và tính toán 


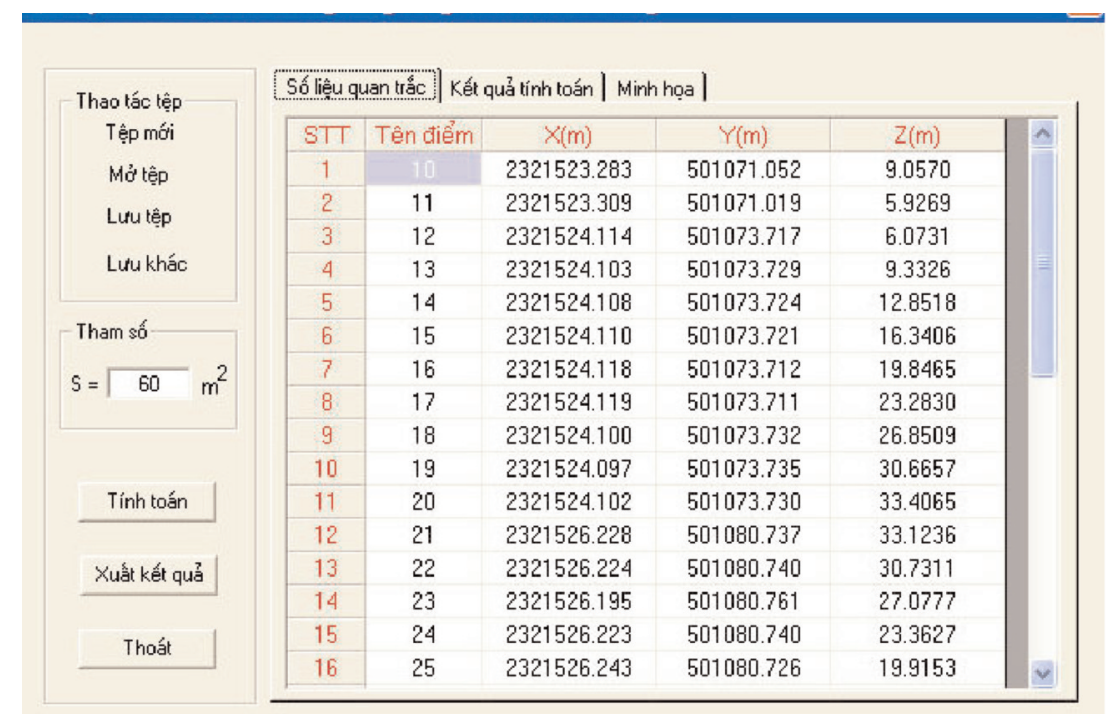

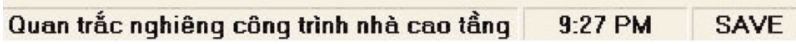

Hình 6: Tệp số liệu đo

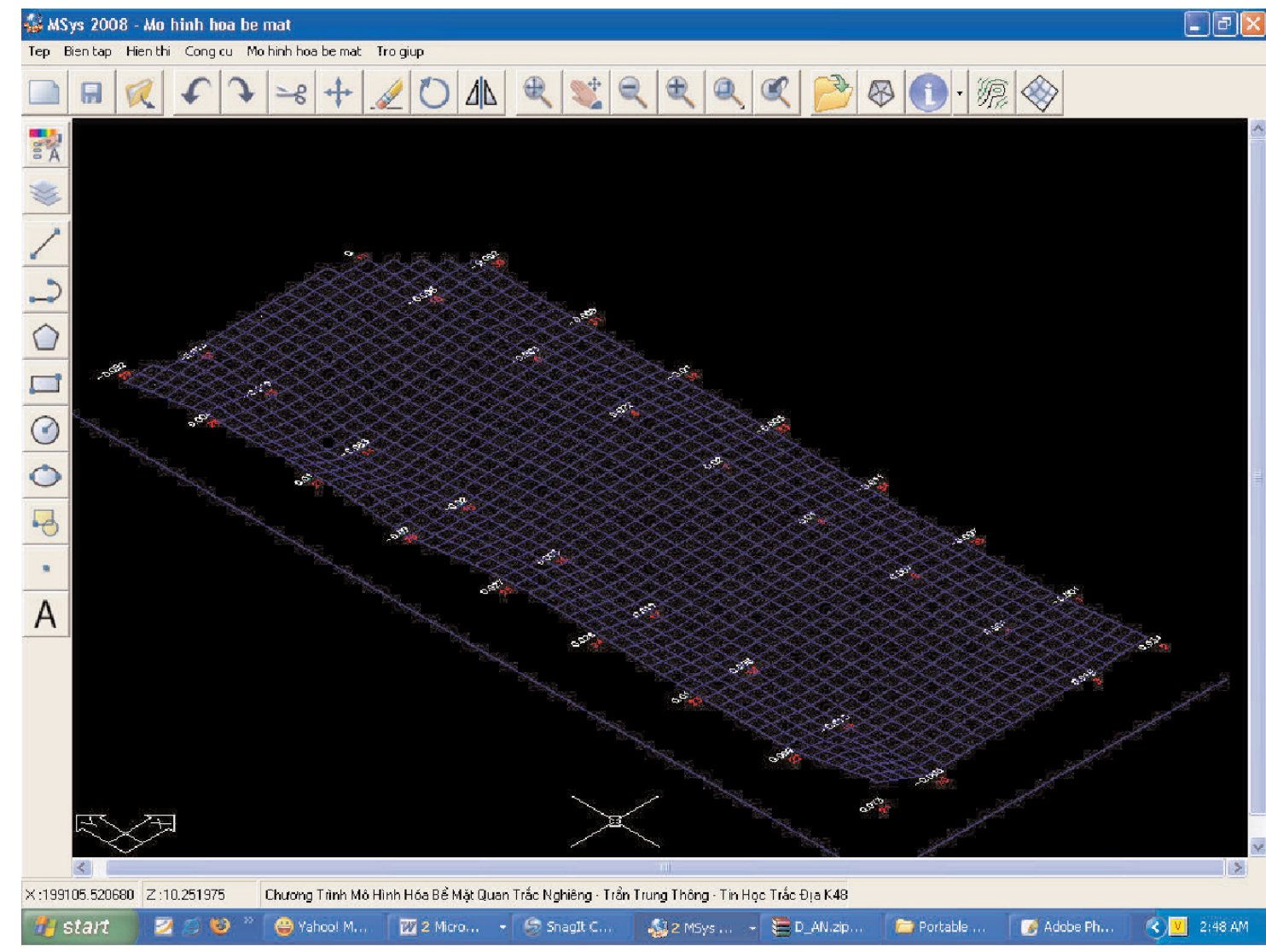

Hình 7: Mô hình bức tường (3D) 


\section{Kết luận}

Các thiết bị đo đạc hiện đại đã mở ra nhiều khả năng mới trong công tác đo đạc nói chung và kiểm tra chất lượng hình học của các công trình. Người làm công tác trắc địa cần khai thác triệt để những ưu điểm của thiết bị đo đạc hiện đại, đồng thời sử dụng công nghệ tin học để xử lý các số liệu quan trắc nhằm thu được kết quả tin cậy. $O$

Tài liệu tham khảo

[1]. Phan Văn Hiến và nnk, 2001. Trắc địa công trình. NXB Giao thông vận tải. Hà Nội, 2001.

[2]. Hoàng Ngọc Hà, Trương Quang Hiếu, 2002. Lý thuyết sai số và phương pháp số bình phương nhỏ nhất. Trường Đại học Mỏ - Địa chất.

[3]. Đỗ Xuân Lôi, 2003. Cấu trúc dữ liệu và giải thuật. NXB Đại học Quốc gia Hà Nội.

[4]. Đinh Công Hòa, 2010. Lập trình bài toán trắc địa cơ sở - NXB Giao thông Vận tải. Hà Nội, 2010.O

Ngày nhận bài 10/4/2013.

ĐỀ XUÂT SỬ DỤNG KINH TUYẾN TRỤC... (Tiếp theo trang 12)

\section{Tài liệu tham khảo}

[1]. Hà Minh Hòa (2008), Bàn về việc xây dựng một số tiêu chuẩn kỹ thuật đo đạc địa chính bằng máy toàn đạc điện tử. Tạp chí địa chính, số 4, tháng 8/2008 - Trang 4 - 10.

[2]. Nguyễn Phi Sơn (2012) "Nghiên cứu xác định độ chính xác đo vẽ bản đồ địa chính các loại đất để hoàn thiện, bổ sung một số quy định trong thành lập bản đồ địa chính ở nước ta". Đề tài $\mathrm{KHCN}$ cấp Bộ, tháng 12/2012.

[3]. Nguyễn Trọng San (2009) Giáo trình "Đo đạc địa chính" dành cho sinh viên ngành địa chính - Đại học Mỏ Địa chất. $\bigcirc$

\section{Summary}

PROPOSED LONGITUDINAL AXIS USING FOR THE DATA PROVINCIAL CADASTRAL UNDER THE ACCURACY OF MAPS FROM THE LAND VALUE

\section{MSc. Nguyen Phi Son - Institute of Geodesy and Cartography}

There are 2 types of distortion of a land parcel's area when projecting from the Earth surface to UTM plane. One of them is distortion caused by the effect from longitudinal projection zone of Geoid to UTM. The second one is distortion caused by measurement errors of each land parcel's vertex. In this paper, we have calculated and compared land parcel's distortion caused by the longitudinal projection zone in each province, in which value were measured with accuracy proposed by a new land price value method. The results show that error caused by distortion of a land parcel's area from the Earth surface to UTM plane is less than that of distortion caused by measurement errors of each land parcel's vertex. Therefore, it is necessary to define 3 degree longitudinal projection zones by uniform province instead of 3 degree zones for each province. $O$

Ngày nhận bài 12/3/2013. 